\title{
Development and installation of a radio frequency quadrupole cooler test
}

Cite as: Rev. Sci. Instrum. 90, 113324 (2019); https://doi.org/10.1063/1.5128225

Submitted: 17 September 2019 . Accepted: 29 October 2019 . Published Online: 26 November 2019

M. Cavenago (D), M. Romé (D), G. Maero, M. Maggiore (D), L. Bellan (D), F. Cavaliere, M. Comunian (D), A. Galatà (D), N. Panzeri, A. Pisent, L. Pranovi, and A. M. Porcellato 


\title{
Development and installation of a radio frequency quadrupole cooler test
}

\author{
Cite as: Rev. Sci. Instrum. 90, 113324 (2019); doi: 10.1063/1.5128225 \\ Submitted: 17 September 2019 • Accepted: 29 October 2019 • \\ Published Online: 26 November 2019
}

M. Cavenago, ${ }^{1, a)}$ (D) M. Romé,, (D) G. Maero, ${ }^{2}$ M. Maggiore,' (D) L. Bellan, ${ }^{1}$ (D) F. Cavaliere, ${ }^{2}$ M. Comunian,

A. Galatà,' (D N. Panzeri, ${ }^{2}$ A. Pisent, ${ }^{1}$ L. Pranovi, ${ }^{1}$ and A. M. Porcellato'

\author{
AFFILIATIONS \\ 'INFN-LNL, Viale dell'Universitá n. 2, 35020 Legnaro (PD), Italy \\ ${ }^{2}$ INFN-MI and Universitá degli Studi di Milano, v. Celoria 16, 20133 Milano, Italy
}

Note: Contributed paper, published as part of the Proceedings of the 18th International Conference on Ion Sources, Lanzhou, China, September 2019.

a) Author to whom correspondence should be addressed: cavenago@Inl.infn.it

\begin{abstract}
A Radio Frequency Quadrupole Cooler (RFQC) prototype was adapted for insertion into a high uniformity magnetic field, with $B_{z}$ up to 0.2 T, to improve radial confinement. While the RFQC purpose is to reduce (by gas collisions) the energy spread and emittance of a beam of radioactive nuclei, to finely select ion mass in nuclear physics, the prototype is tested in a setup including a stable ion source, a pepper pot emittance meter, and two Faraday cups; this makes a precise characterization of the RFQC feasible. The ion extraction was studied in detail by simulations, both to match it to the emittance meter granularity and to verify the effect of the typical nonuniformity of the longitudinal electric field $E_{z}$ inside the RFQC; an average motion description (including friction force from gas collisions) was used, introducing the ballistic and diffusive regimes. With a preliminary optimization of the electrode shape, buffer gas pressure $p_{g}$, and radio frequency voltage, the ion beam can be extracted with a significant cooling margin.
\end{abstract}

Published under license by AIP Publishing. https://doi.org/10.1063/1.5128225

\section{INTRODUCTION}

For the accurate mass spectrometry (with resolution goal 1:20 000) of exotic ion beams, ${ }^{1}$ it is necessary to cool ions both in energy spread (goal is about $0.5 \mathrm{eV}$ rms or better) and in transverse oscillations. ${ }^{2}$ In the radio frequency (rf) quadrupole cooler (RFQC), this is accomplished by collisions with a light gas, while ions are trapped and transported by $\mathrm{rf}$ and static voltages applied to RFQC electrodes.

The actual performance depends on a fine balance between cooling and heating effects (due to collisions and rf field) and on the ion extraction process, which needs a complicated theoretical, numerical, and experimental investigation. ${ }^{3-5}$ To this aim, a prototype RFQC was developed at INFN-LNL and INFN-MI with a ten-fold longitudinal segmentation of electrodes and an external axial magnetic field (provided by the Eltrap device's solenoid ${ }^{6}$ ) to improve confinement at reduced rf voltage; Eltrap stands for "Electron Trap." This installation into the Eltrap facility has also the practical purpose to test the diagnostics (emittance meters) and to train for the operation and maintenance of another RFQC, ${ }^{8}$ to be installed at the SPES (Selective Production of Exotic Species) accelerator of LNL, where exotic nuclei produced by a primary source will be selected and reaccelerated; ${ }^{1}$ some emphasis is given to nuclei with atomic number $A \in[80,140]$.

Note that the ion kinetic energy (before deceleration at RFQC input) $K_{1}$ is relatively large $(40 \mathrm{keV})$ in the SPES installation, being determined by the primary source. Furthermore, a larger ion kinetic energy $K_{2} \geq 100 \mathrm{keV}$ may be required at the input flange of the high resolution mass spectrometer (HRMS); thus, HRMS may be placed on some high voltage (HV) platform ${ }^{2}$ [see Fig. 1(a)]. On the contrary, in the Eltrap installation, $K_{1}$ is limited by the installed $\mathrm{Cs}^{+}$ ion source to $5 \mathrm{keV}$ or less; for simplicity, we treat only the case $K_{1}=5 \mathrm{keV}$ in the following results, noting that all voltages have to be scaled down or adjusted when $K_{1}$ is smaller. With the Eltrap vacuum chamber as the ground reference, the ion source emitter is held at $V_{s}=200 \mathrm{~V}$ so that the injection line drift tubes are at $V_{0}=V_{2}=V_{4}=V_{s}-K_{1} / e=-4.8 \mathrm{kV}$ (see Ref. 7 for notation and Fig. 1 therein for an overall scheme; in brief, injection line electrodes 
(a)

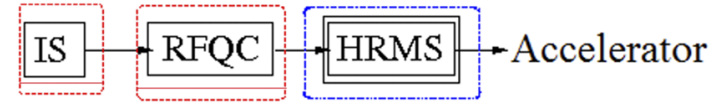

(b)

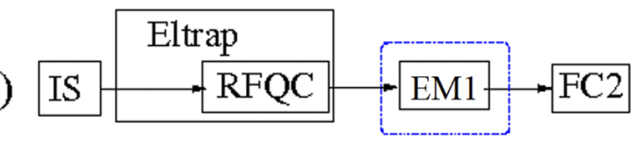

Positive HVP

Negative HVP

FIG. 1. High Voltage Platforms (HVPs) for ion sources (IS) and RFQC setups: (a) RFQC before HRMS; (b) RFQC in Eltrap.

are labeled from $\mathrm{V} 0$ to $\mathrm{V} 7$ and extraction electrodes from $\mathrm{V} 8$ to $\mathrm{V} 11$, held at voltages $V_{i}$ with $\left.i=0, \ldots, 11\right)$. This ground choice simplifies ion energy loss measurement (see later) and RFQC control (possibly with some complications in ion source management), so it is adequate for a test installation as the present one. The whole beamline fits $^{7}$ inside the Eltrap solenoid, which can provide a magnetic flux density component $B_{z}$ up to $0.2 \mathrm{~T}$; the purpose is to investigate possible advantages ${ }^{9}$ and disadvantages of $B_{z}$ with an existing solenoid, before finalizing design to an accelerator installation (and possibly higher $B_{z}$ ).

The injection line including a Faraday cup FC1 (see Fig. 2) and the RFQC enclosure (which is needed to regulate and maintain a He gas pressure up to $10 \mathrm{~Pa}$ ) were indeed mounted on a rigid support for insertion into Eltrap; the rf matching box was built, ${ }^{10}$ and the in-vacuum multiplexer (for the distribution of $\mathrm{rf}$ and static voltages to the electrodes) is in the assembly stage. At the same time, the test $\mathrm{Cs}^{+}$ion source and a movable pepper pot emittance meter were calibrated in a test bench.

Another issue is the challenging accuracy (sub-electronvolt) of the output energy measurement: ${ }^{1,12}$ the stability of the bias $V_{d}$ between the RFQC and the source should be in the order of $0.1 \mathrm{~V} \mathrm{rms}$; finally, a device like a Retarding Field Energy Analyzer with similar accuracy ${ }^{11}$ must be used and adapted, which also requires time consuming simulations. To this aim, another Faraday cup FC2 with retarding field and grids is under construction.

The rest of this paper is organized as follows: Sec. II introduces the Eltrap extraction beamline, with some setup details.

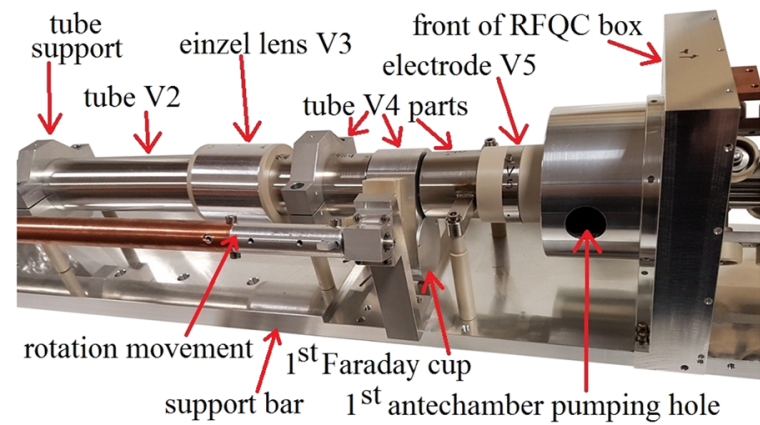

FIG. 2. Injection line into the RFQC (box cover removed); note the movement for the 1st Faraday cup.
Section III gives a simplified description of the RFQC beam transport, making use of the ponderomotive potential concept, including the $\mathrm{Cs}^{+} / \mathrm{He}$ cross section and introducing the distinction between ballistic and diffusive regimes in the ion drift; the potential distribution at junctions between RFQC segments is also evaluated. The last section reports ballistic beam simulations, with the aim of validating the electrode shape of the extraction line; a good matching of the beam to the emittance meter physical resolution limits is obtained.

\section{SETUP}

The $\mathrm{Cs}^{+}$ion source (maximum ion energy $5 \mathrm{keV}$ ) and the emittance meter were commissioned in a small test bench built around a CF160 6-way cross (see Figs. 1 and 2 in Ref. 13), up to a few hundreds of nanoampere current. A first matching box, ${ }^{10}$ also splitting the rf input into two rf outputs (in phase opposition) matched to the RFQC load (mainly capacitive, $200 \mathrm{pF}$ ), was built; the operation frequency is $4 \mathrm{MHz}$.

The RFQC enclosure is a $0.74 \mathrm{~m}$ long box (gas tight but for the necessary beam passage and connections), mounted together with the injection line and source on a rigid $1.72 \mathrm{~m}$ long $\mathrm{Al} /$ stainless steel bar (partly shown in Fig. 2); the box is earthed, and all RFQC section static voltages $V_{i}^{s}$ are roughly within the range $\left[0, V_{s}\right]$, which allows us to reuse the Eltrap power supplies. ${ }^{6}$ On the contrary, the ion source and emittance meter have to be powered by a HV platform at $V_{0}=-4.8 \mathrm{kV}$ potential.

In our coordinate system, $z$ is the beam axis (horizontal), $z=0$ is the Eltrap yoke middle, $y$ is the vertical axis, and the source emitter is placed at $z_{s}=1.02 \mathrm{~m}$. The beam is directed toward negative $z$, so the pepper pot emittance meter EM1 can be inserted at $z=-1.05 \mathrm{~m}$. A Faraday cup FC1 can be inserted at $z=0.09 \mathrm{~m}$ into the V4 drift tube to check RFQC input current; a second cup FC2 (under construction) will be placed downstream of EM1, primarily to verify $\mathrm{Cs}^{+}$ transmission.

Moreover, by scanning FC2 collector and suppression voltages, a simplified RFEA (retarding field energy analyzer ${ }^{11}$ ) can be

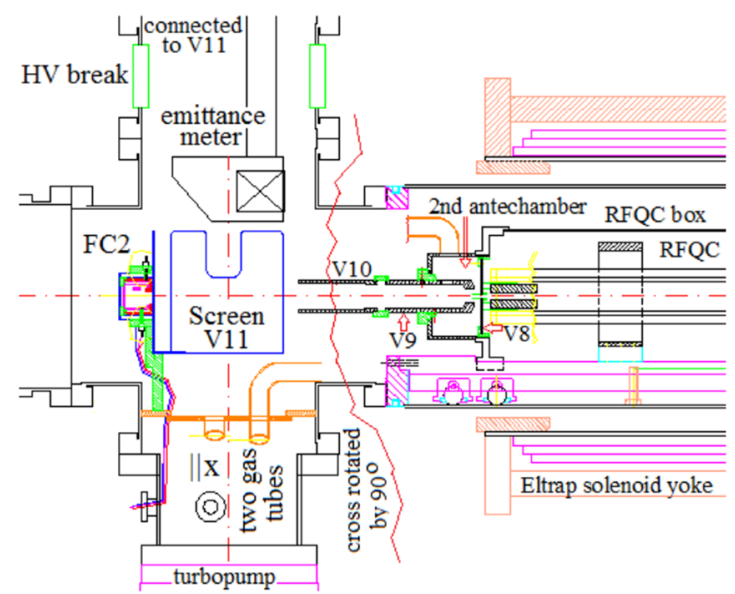

FIG. 3. Details of the extraction line (with emittance meter retracted and part of gas tubes shown). 
implemented to verify the energy spread. Achieving a subelectronvolt resolution is challenging and may require a well focused beam or fine pitch grids. ${ }^{11,12}$ Similarly, by changing $V_{s}$ by $\pm \Delta V$, we can mimic a larger input energy spread, increased by an amount proportional to $e \Delta V$ (with usual rules for summing rms values; for example, a source energy spread of $0.1 \mathrm{eV} \mathrm{rms}$ and $\Delta V=5 \mathrm{~V}$ give a spread of $4.1 \mathrm{eV} \mathrm{rms}$ ); electronic noise sums similarly.

The extraction beamline is made of two parts, see Fig. 3: one part (including a gas pumping antechamber, extraction electrode V8, and drift tubes V9 and V10) is cantilevered on the RFQC box and the other (including the Faraday cup FC2 and the screen preceding it) is bolted to the vacuum chamber.

\section{ION TRANSPORT}

Let $m_{i}$ and $q=e$ be the ion mass and charge, with $K_{i}=\frac{1}{2} m_{i} v^{2}$ the kinetic energy and $\mathbf{v}$ the ion velocity in the laboratory frame; similarly, let $m_{t}$ be the mass of one gas molecule (called target). Since $m_{t} / m_{i} \ll 1$ in our case (He target, $\mathrm{Cs}^{+}$ion), the ion trajectory is not largely perturbed by each single gas-Cs collision; after averaging on all collisions, the first order effect is a friction force $\mathbf{F}_{r}$ (at the second order, we have Cs diffusion and straggling, whose details are outside this paper's scope; heavier gases will increase these $\left(m_{t} / m_{i}\right)^{2}$ effects and are similarly postponed to future studies). Also, the static electric field $\mathbf{E}=-\nabla \phi$ and the ponderomotive force $\left(\mathbf{F}_{p}\right)$ contribute to driving the ion macromotion (motion average on a rf period); the averaged total force is thus

$$
\mathbf{F}=\mathbf{F}_{r}+q \mathbf{E}+q \mathbf{v} \times \mathbf{B}+\mathbf{F}_{p}, \quad \mathbf{F}_{p}=-q \nabla \phi_{p} \equiv q \mathbf{E}_{p},
$$

where $\phi_{p}$ is the so-called ponderomotive potential. ${ }^{7}$ Its value $V_{p}$ $=\frac{1}{2} m\left(\omega_{M} r_{0}\right)^{2} / e$ at $r=r_{0}$ (on RFQ rods) is related to the macromotion angular frequency,

$$
\omega_{M} \equiv \frac{k_{q} \omega}{\sqrt{8}}, \quad k_{q}=\frac{\left|4 e V_{r f}\right|}{m_{i} \omega^{2} r_{0}^{2}},
$$

where $k_{q}$ is the stability parameter of Mathieu equations for micromotion, ${ }^{14} \omega$ is the $\mathrm{rf}$ angular frequency, $2 V_{r f}$ is the $\mathrm{rf}$ peak to peak voltage, and $2 r_{0}$ is the distance between RFQ opposing rods. The micromotion stability condition ${ }^{9}$ is $k_{q}<0.908-O\left(\Omega_{i}^{2} / \omega^{2}\right)$ where $\Omega_{i}$ is the ion cyclotron angular frequency; for example, with $\omega / 2 \pi=4 \mathrm{MHz}, r_{0}=4.5 \mathrm{~mm}$, and $V_{r f}=200 \mathrm{~V}$, we have $k_{q}=0.045$ and $\Omega_{i} / \omega<0.01$ with a large safety margin.

Note that the friction force depends on the difference between the ion and average gas flow velocities; the latter is usually negligible, for gas flow is partly impeded by the electrode system itself; in other words, the gas is at rest in the laboratory frame, and the friction force takes the form $\mathbf{F}_{r}=-m_{i} \mathbf{v} v_{m}\left(K_{i}\right)$ where $v_{m}$ is the momentum collision frequency. From the $\mathrm{Cs}^{+}-\mathrm{He}$ interatomic potential ${ }^{3}$ and standard collision kinematics, $v_{m}$ can be easily calculated averaging on all impact parameters, with the result

$$
v_{m}=f_{1} n_{g}|v| \sigma\left(K_{i}\right), \quad f_{1}=\frac{m_{t}}{m_{t}+m_{i}}, \quad \sigma=\pi r_{e}^{2},
$$

where $n_{g}$ is the gas density, $\sigma$ is the cross section, and $r_{e}\left(K_{i}\right)$ is the effective collision radius. The mobility coefficient $\mu\left(K_{i}\right) \equiv q /\left(m_{i} v_{m}\right)$ is related to the equilibrium velocity $\mathbf{v}_{e q}$ for a hypothetical $F=0$ case with uniform electric field and $\mathbf{B}=0$; thanks to Eq. (1), this is

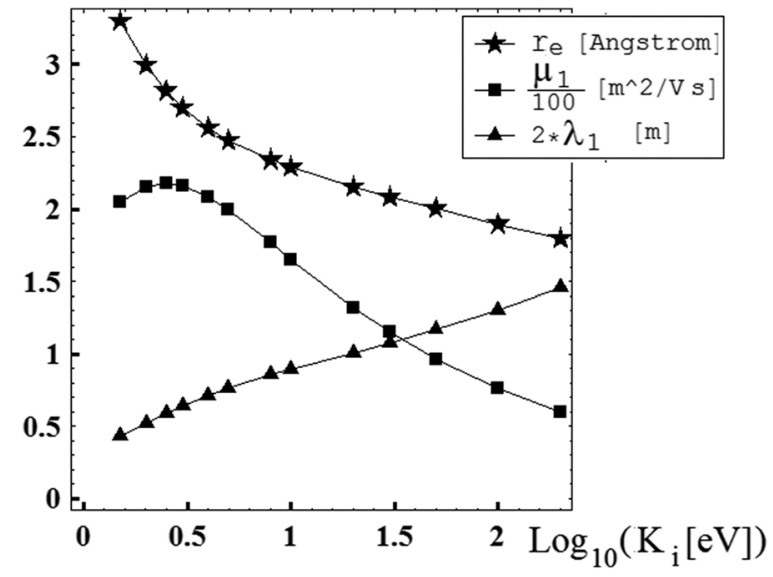

FIG. 4. Collision radius for $\mathrm{Cs}^{+}$against $\mathrm{He}$ (at rest) vs $\mathrm{Cs}^{+}$kinetic energy $K_{i}$ from $1.5 \mathrm{eV}$ to $200 \mathrm{eV}$ [also, $\lambda_{1}$ as defined after Eq. (4) and $\mu_{1}$ are shown]; see legend for units.

$\mathbf{v}_{e q}=\mu\left(K_{i}\right)\left[\mathbf{E}+\mathbf{E}_{p}\right]$. In general, the friction force may also be written as $\mathbf{F}_{r}=-e \mathbf{v} / \mu$.

Let $\mu_{1}$ be the $\mu$ value when $p_{g}=1 \mathrm{~Pa}$; of course, $\mu=\mu_{1} / p_{g}$ for other He pressures $p_{g}$ expressed in Pascal. We plot $r_{e}$ and $\mu_{1}$ in Fig. 4; note that $r_{e}$ decreases only slowly with $K_{i}$ (similarly to the hard sphere approximation, where $r_{e}$ is constant); also, $\mu_{1}$ decreases slightly with $K_{i}$ due to the opposing increase of $|v|$ and decrease of $r_{e}$. For rapid ion tracing, we take $\mu_{1} \cong 150 \mathrm{~m}^{2} / \mathrm{V} \mathrm{s}$; this is similar to the value of $200 \mathrm{~m}^{2} / \mathrm{V}$ s used in Ref. 7 .

The decrease of ion kinetic energy along the beam path $s=-z$ is similarly calculated as

$$
\frac{\partial K_{i}}{\partial s}=-\frac{K_{i}}{\lambda_{e}}, \quad \frac{1}{\lambda_{e}}=n_{g} \sigma f_{2}, \quad f_{2}=\frac{2 m_{t} m_{i}}{\left(m_{t}+m_{i}\right)^{2}},
$$

with $\lambda_{e}$ a kind of attenuation distance for ion energy; its value $\lambda_{1}$ for $n_{g}=2.4 \times 10^{20} \mathrm{~m}^{-3}$ (that is at pressure $p_{g}=1 \mathrm{~Pa}$ ) is also given in Fig. 4; moreover, $\lambda_{e}=\lambda_{1} / p_{g}$.

In most RFQCs, due to the electrode segmentation as in our case, the on-axis electric potential $\phi(0,0, z)$ has not an uniform slope, but as in a stairway, it changes from one plateau (or step) to the next (see Fig. 5), with jumps of half width $g$ (given later), spaced by distances $L_{s}$, equal to the electrode periodicity or length; in our case, $L_{s}$ $=72 \mathrm{~mm}$. Electrodes and on-axis potential are also described in Refs. 3-5 and 15 .

If $p_{g} \ll 1 \mathrm{~Pa}$, we have $\lambda_{e} \gg L_{s}$ for each step, so obtaining a negligible cooling. For $p_{g} \cong 4 \mathrm{~Pa}$, we have $\lambda_{e} \cong 150 \mathrm{~mm}$ at $K_{i}=100 \mathrm{eV}$, so two steps are enough for a considerable reduction of energy; still, we have $\lambda_{e}>L_{s}$, which we define as the ballistic regime, where the ion rapidly passes through the plateau, reaching the potential jump where its energy $K_{i}$ is increased again (by $10 \mathrm{eV}$ in Fig. 5 case). In the opposite case of diffusive regime $\lambda_{e} \ll L_{s}$, the ion is nearly stopped inside one plateau and completes its travel to the next jump helped by diffusion. The latter seems helpful for cooling, but the long residence time raises concerns about transverse losses; in our RFQC, voltages are graded so that the ion path is in the ballistic regime, with possibly the step before extraction approaching the diffusive regime. 


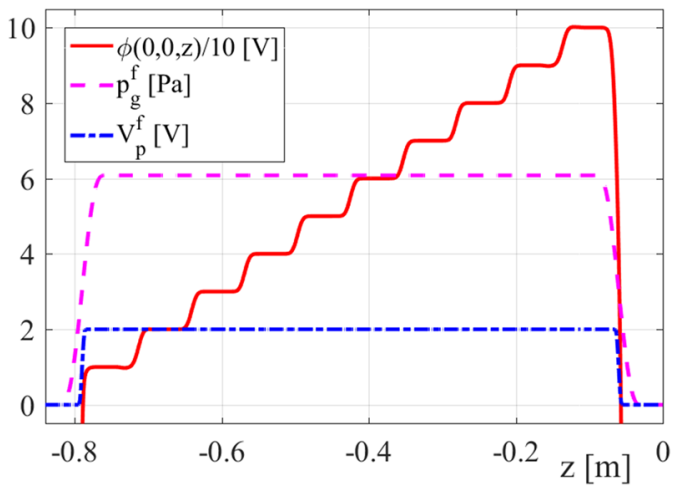

FIG. 5. Profile of on-axis potential (divided by 10) vs $z$; also, $p_{g}^{f}$ and $V_{p}^{f}$ are shown (see legend for units).

A practical reason to avoid purely diffusive regimes is the large $p_{g}$ required (which makes installing a reasonably effective differential pumping difficult). In the beam simulations, we will use Eq. (1), and thus, we neglect diffusive effects, which is well justified for most of these RFQCs.

Let us now calculate the major effects of an RFQC electrode segmentation in detail. The on-axis potential for two cylinder electrodes (with inner radius $R$ and a negligible gap in between, held for simplicity at potentials $\pm V_{0}$ ) can be well approximated ${ }^{16}$ by $V_{0} \tanh \left[z^{\prime} /(2 g)\right]$, where $g \cong R / 2.64$ and $z^{\prime}$ is a local coordinate with $z^{\prime}=0$ the middle plane between electrodes; in the simplified 3D electrode geometry, in place of each cylinder, we have four rods (circumscribed to a $R=r_{0}=4.5 \mathrm{~mm}$ construction cylinder) at the static potential $\phi=-V_{0}$ for $z^{\prime}<0$ and $V_{0}$ for $z^{\prime}>0$; also, a $\phi=0$ overall enclosure at $R_{1}=31 \mathrm{~mm}$ accounts for RFQC support bars. In Fig. 6 results, we note that (1) the onaxis potential has the same fit form, with a similar $g=2.05 \mathrm{~mm}$ $\cong r_{0} / 2.2$ half width, and (2) the off-axis potential does not appreciably depend on azimuth $\arctan (y / x)$ at least for $r \leq 0.6 R$, that is to say, multipoles (octupole and harmonics) in static potential are well negligible.

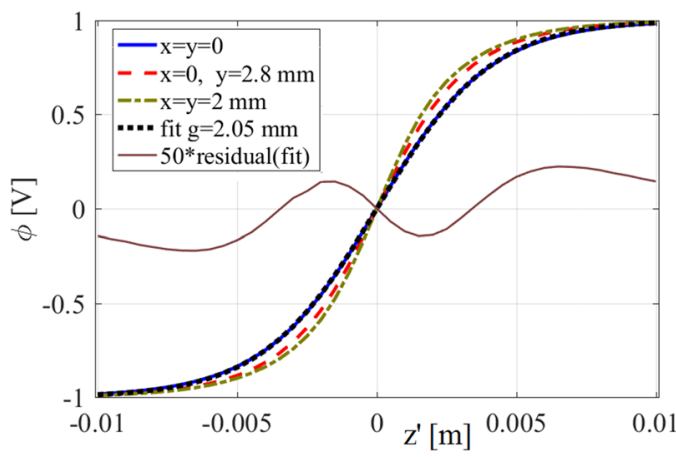

FIG. 6. Profiles of the static potential (which controls drift) at a segmentation of electrodes (held at $-V_{0}$ and $V_{0}=1 \mathrm{~V}$ ) from $3 \mathrm{D}$ simulations.

\section{EXTRACTION BEAMLINE OPTIMIZATION}

As is well known, unipolar electrostatic lenses are always converging, while immersion lenses (or anode lens) may be defocusing when the beam is decelerated; a focusing strength index is $Q(z)=3\left(V_{a}^{\prime}(z) / V_{a}(z)\right)^{2} / 16$ with $V_{a}=V_{s}-\phi(0,0, z)$ the acceleration potential. ${ }^{16}$ So in the injection beamline design, ${ }^{7}$ the main concern was to balance the defocusing effect of the necessary beam deceleration, by converging lenses, provided by a four-electrode system. The gas conductance was kept as low as possible, and lateral holes for differential gas pumping were provided. The extraction design is seemingly simpler, since beam acceleration gives focusing; but actually a too strong focusing should be avoided (since it gives large divergences later). Telescopic systems, named "triode" (3 electrodes) or "tetrode" (4 electrodes), were so envisioned, with a converging lens after the waist that is unavoidably formed after beam reacceleration. The extraction design is also constrained by practical considerations: (1) the same gas flow issue as in the injection; (2) until it reaches the emittance meter EM1 at potential $V_{11}$ (from -2 to $-4.8 \mathrm{kV}$ ), the beam should be shielded from the vacuum chamber potential by some set of drift tubes held at potential $V_{10} \cong V_{11}$; when EM1 is retracted, a surrounding screen remains in place and shields the beam up to FC2; (3) the EM1 acceptance is $r<R_{1}=12.5 \mathrm{~mm}$, and divergence is within $\pm 12 \mathrm{mrad}$; the beam should be contained inside these limits with large safety margins (say factor 2 to account for possible fluctuations of RFQC performance); on the other hand, the beam radius must exceed pepper spacing $L_{p}=1 \mathrm{~mm}$.

The ion trajectories were simulated with an adaptive step leapfrog, using Eq. (1) and the Eltrap $B_{z}$ profile ${ }^{6}$ with $B_{z}=0.11 \mathrm{~T}$ at the solenoid center; the gas pressure profile $p_{g}^{f}(z)$ is zero outside the range $[-0.82,-0.03] \mathrm{m}$ and has smooth transitions $(6 \mathrm{~cm}$ wide) to the constant $p_{g}$ inside this range (see Fig. 5). The ponderomotive potential $V_{p}^{f}(z)$ is zero outside the range $\left[z_{1}-w, z_{2}+w\right]$ and smoothly rises to $V_{p}$ in a length $2_{w}=1 \mathrm{~cm}$, as $V_{p}^{f}(z)=V_{p} \Theta_{s}((z$ $\left.\left.-z_{1}\right) / w\right) \Theta_{s}\left(\left(z_{2}-z\right) / w\right)$ with $z_{1}=-0.785 \mathrm{~m}$ and $z_{2}=-0.065 \mathrm{~m}$ and $\Theta_{s}$ is a smoothed Heaviside function; this range roughly follows the RFQ box extents. In detail, $\Theta_{s}(x)=\left[8+\left(15-10 x^{2}+3 x^{4}\right) x\right] / 16$ for $|x|<1$ and $\Theta_{s}(x)$ equals the Heaviside function elsewhere.

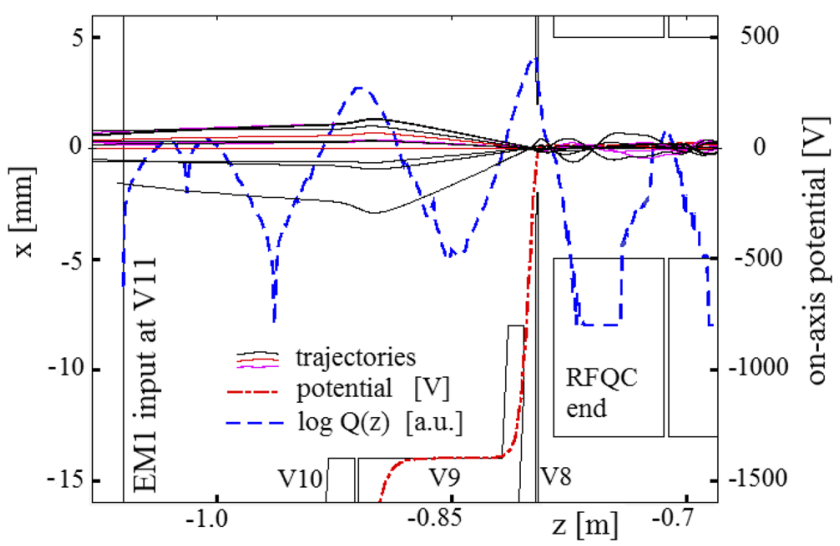

FIG. 7. Plot of rays in the extraction design (thin lines); for comparison, $\phi(0,0, z)$ and the focusing strength index $Q(z)$ are shown (thicker lines). 

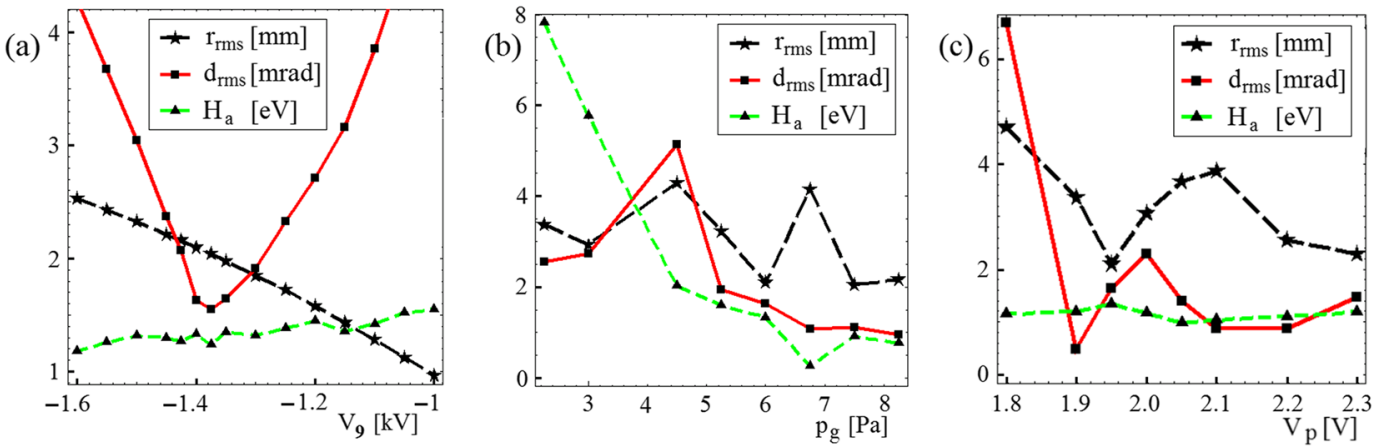

FIG. 8. (a) Final (at the emittance meter) rms radius $r_{\text {rms }}$ and divergence $d_{\text {rms }}$ vs electrode voltage $V_{9}$ with $p_{g}=6 \mathrm{~Pa}$; the final average energy $H_{a}$ (about $13 \pm 2 \mathrm{eV}$ ) is also shown; (b) same quantities vs gas pressure $p_{g}$, with $V_{9}=-1.4 \mathrm{kV}$ and $V_{p}=1.95 \mathrm{~V}$; (c) same quantities vs $V_{p}$ with $p_{g}=6 \mathrm{~Pa}$ and $V_{9}=-1.4 \mathrm{kV}$.

Ion tracing starts at $z=z_{s}=0.986 \mathrm{~m}$ (ion source waist) before entering the solenoid field, as in Ref. 7. Due to simplified model axial symmetry, ion rays start from the $x x^{\prime}$ plane, that is, $y\left(z_{s}\right)=0=y^{\prime}\left(z_{s}\right)$, filling (uniformly as possible) an ellipse with $x$-semiaxis $x_{1}=2 \mathrm{~mm}$ that is the assigned waist envelope and $x^{\prime}$-semiaxis $d_{1}=5 \mathrm{mrad}$ that is the source divergence; only rays with $x>0$ are traced, and results for uniformly distributed azimuth $\arctan (y / x)$ are calculated only in postprocessing (error $\pm 15 \%$ ).

For the triode system, see the overall trajectory plot in Fig. 7 and a scan of the electrode voltage $V_{9}$ for the case $p_{g}=6 \mathrm{~Pa}$ and $V_{p}=1.95 \mathrm{~V}$ in Fig. $8(\mathrm{a})$; the voltage $V_{9} \cong-1.4 \mathrm{kV}$ is preferable since it attains a clear divergence minimum, while it provides a beam radius convenient for both considerations explained. The residual average total energy $H_{a}$ at EM1 $(13 \mathrm{eV}$, similar to the voltage jump of $10 \mathrm{~V}$ ) compared to the total ion energy $H_{i}$ at the source $(200 \mathrm{eV})$ shows that $6 \mathrm{~Pa}$ provides enough friction. Indeed, Fig. 8(b) shows that at lower $p_{g}$, the residual energy is considerably larger $(70 \mathrm{eV})$. At $6 \mathrm{~Pa}$, for a $5 \mathrm{eV}$ input $H_{i}$ change, $H_{a}$ changes by $0.7 \mathrm{eV}$, which indicates a lower bound for the output energy spread; this is a promising step for reaching a $1 \mathrm{eV}$ pp output spread, even if worthwhile for further investigation and optimization. The range of $V_{p}$ [in Fig. 8(c)] is rather small as a lower $V_{p}$ gives less confinement (simulation is stopped if an ion collides with any electrode) and a larger $V_{p}$ induces more scalloping of the beam inside the RFQC (with possibly some preferred phase of scallop oscillation for extraction).

\section{CONCLUSION AND PERSPECTIVES}

A test facility for an RFQC is being completed, featuring several beam input/output diagnostics and the possibility of adding a $B_{z}$ field. The extraction beam optics was adapted to the existing pepper pot emittance meter, while the energy spread diagnostic is under development. The effects of RFQC electrode segmentation were theoretically assessed and introduced in simulations, which need to account for a complicated geometry, now including the extraction optics; we plan to improve computational efficiency in order to include diffusion and beam straggling. A differential gas pumping system is also in completion.

\section{REFERENCES}

${ }^{1}$ A. Andrighetto, L. Biasetto, M. Manzolaro et al., AIP Conf. Proc. 1099, 728 (2009).

${ }^{2}$ M. Comunian, C. Baltador, L. Bellan, M. Cavenago, A. Pisent, and E. Khabibullina, "Design of high resolution mass spectrometer for SPES," in 9th International Particle Accelerator Conference (IPAC18), Vancouver, 2018, http://www.jacow.org, pp. 3252-3254.

${ }^{3}$ S. Schwarz, Nucl. Instrum. Methods Phys. Res., Sect. A 566, 233 (2006).

${ }^{4}$ F. Herfurth, J. Dilling, A. Kellerbauer et al., Nucl. Instrum. Methods Phys. Res., Sect. A 469, 254 (2001).

${ }^{5}$ B. R. Barquest, F. Ames, T. Au et al., "Development and installation of the CanREB RFQ Buncher at TRIUMF," in 9th International Particle Accelerator Conference (IPAC18), Vancouver, 2018, http://www.jacow.org, pp. 3914-3916.

${ }^{6}$ M. Amoretti, G. Bettega, F. Cavaliere, M. Cavenago, F. De Luca, R. Pozzoli, and M. Romé, Rev. Sci. Instrum. 74, 3991 (2003).

${ }^{7}$ M. Cavenago, M. Romé, M. Maggiore, A. M. Porcellato, G. Maero, F. Chiurlotto, M. Comunian, A. Galatà, and F. Cavaliere, Rev. Sci. Instrum. 87, 02B504 (2016).

${ }^{8}$ R. Boussaid, G. Ban, G. Quéméner, Y. Merrer, and J. Lorry, Phys. Rev. Accel. Beams 20, 124701 (2017).

${ }^{9}$ G.-Z. Li and G. Werth, Phys. Scr. 46, 587 (1992).

${ }^{10}$ M. Cavenago, L. Bellan, M. Comunian, M. Maggiore, L. Pranovi, G. Maero, N. Panzeri, and M. Romé, "A RFQ cooler development," in 9th International Particle Accelerator Conference (IPAC18), Vancouver, 2018, http://www.jacow.org, pp. 3626-3630.

${ }^{11}$ M. Cavenago, L. Bellan, and M. Comunian, AIP Adv. 8, 125221 (2018).

${ }^{12}$ Y. Cui, Y. Zou, A. Valfells, M. Reiser, M. Walter, I. Haber, R. A. Kishek, S. Bernal, and P. G. O'Shea, Rev. Sci. Instrum. 75, 2736 (2004).

${ }^{13}$ M. Cavenago, M. Romé, G. Maero, M. Maggiore, L. Bellan, M. Comunian, and L. Pranovi, "Development of a combined RFQ cooler with axial magnetic field," in LNL Annual Report 2018, 2019, p. 198, available at http://www.lnl.infn.it/ annrep/read_ar/2018.

${ }^{14}$ R. B. Moore, O. Gianfrancesco, R. Lumbo, and S. Schwarz, Int. J. Mass Spectrom. 251, 190 (2006).

${ }^{15}$ C. M. Ricketts, B. S. Cooper, G. Edwards et al., Nucl. Instrum. Methods Phys. Res., Sect. B (in press).

${ }^{16}$ A. B. El-Kareh and J. C. J. El-Kareh, Electron Beams, Lenses, and Optics, Especially (Academic Press, New York, 1970), pp. 62-68, Eq. (4.39). 